\title{
GI-COVID: Are There COVID-19 Patients with Primary Gastrointestinal SARS-CoV-2 Infection and Symptoms?
}

\author{
John Ong ${ }^{1,2} \cdot$ Yock Young Dan ${ }^{1,3}$ \\ Accepted: 4 December 2020 / Published online: 11 January 2021 \\ (c) The Author(s), under exclusive licence to Springer Science+Business Media, LLC part of Springer Nature 2021
}

Keywords Gastroenterology · Gastrointestinal · COVID-19 · SARS-CoV2 · Coronavirus

Since its first outbreak in late 2019, the severe acute respiratory syndrome coronavirus-2 (SARS-CoV-2) has spread rapidly, ravaging industries and economies and claiming over a million lives worldwide. Approximately 1 year later, much has been learnt about this 29.9 kilobase pair, positive-sense, single-stranded RNA virus (ssRNA +). It is now understood that a lipid bilayer envelops the SARS-CoV-2 RNA and that its genome encodes the non-structural proteins (nsps) 1a and $1 \mathrm{~b}$, the trimeric glycoprotein spikes (S), the membrane proteins $(\mathrm{M})$, the envelop proteins (E), and the nucleocapsid proteins $(\mathrm{N})$. $\mathrm{S}$ is primed by host transmembrane serine protease 2 (TMPRSS2), and after its cleavage, the receptor binding domain (RBD) on the $\mathrm{S} 1$ subunit binds directly to the peptidase domain on angiotensin-converting enzyme 2 receptors (ACE2) in order to enable entry of the virus into host cells [1]. A D614G mutation in the S1 subunit has since led to a more virulent strain that is responsible for most COVID-19 cases worldwide. The S2 subunit assists with the fusion of the virus to the cell membrane, and once within the host cell, the E protein forms a cation-selective channel across the Endoscopic Reticulum-Golgi Intermediate Compartment (ERGIC) membrane, which plays an important role in the pathogenicity of SARS-CoV-2; the E protein is potentially a target for drug therapy [2]. Briefly, the end result is that the virus hijacks intracellular machinery in order to produce multiple copies of itself, subsequently triggering a deleterious cascade, including caspase- 8 activation leading

John Ong

jo401@cam.ac.uk

1 Department of Medicine, National University of Singapore, Singapore, Singapore

2 Department of Engineering, University of Cambridge, Trumpington Street, Cambridge CB2 1PZ, UK

3 Department of Gastroenterology and Hepatology, National University Hospital, Singapore, Singapore to cell death and tissue inflammation $[3,4]$. Therefore, the distribution of ACE2 in tissue importantly influences disease symptomatology and pathophysiology.

Since ACE2 is expressed in the respiratory tract including the nasal and oral mucosa, COVID-19 typically manifests with a cluster of symptoms that include fever, cough, anosmia, and dysgeusia. Nevertheless, ACE2 is significantly more abundant in the gastrointestinal (GI) tract compared with the lungs (www.proteinatlas.org) although the pattern of distribution varies; high ACE2 expression is present in the stomach and small bowel in contrast to comparatively lower levels of ACE2 expression in esophagus, liver, and colon [5, 6]. The varying expression and distribution of ACE2 within the GI tract may explain to a degree the GI symptoms experienced by COVID-19 patients. For example, a recent study reported that GI hemorrhage in COVID-19 patients resulted mainly from ulcers in areas of high ACE2 expression within the GI tract, in particular the stomach and the duodenum [7]. The presence of the SARS-CoV-2 virus in gastric, duodenal, and rectal tissue in the context of GI bleeding has previously been reported [6]. In addition, fecal calprotectin, a reliable marker of enteric inflammation, is elevated in some COVID19 patients with GI symptoms [8].

With regard to GI symptomatology, recent large systematic reviews and meta-analyses have established that the most commonly reported GI symptoms in COVID-19 patients are anorexia (15.8-26.8\%), diarrhea (7.7-12.5\%), nausea and vomiting $(6.0-10.2 \%)$, jaundice (6.0-16.7\%), and abdominal pain (3.0-9.2\%) [9-11]. It is noteworthy that these are the reported pooled prevalence rates of observational studies that involved predominantly hospitalized COVID-19 patients, a population that may misrepresent all COVID-19 patients. Significant heterogeneity between studies was also detected in meta-analyses, including geographical variation on subgroup analyses. Therefore, clinicians may find that the frequency of GI symptoms in COVID-19 
differs significantly from these estimates in their local population. Though GI bleeding, which was not included in the studies cited above, has been reported in $6.3-19.2 \%$ of hospitalized COVID-19 patients, however the study populations were small $(n<200)[6,12,13]$. Nonetheless, it is becoming increasingly clear that there is a subgroup of patients who experience GI symptoms alone without any respiratory symptoms $[9,10]$. Hereon, this phenomenon is referred to as GI-COVID.

Based on a retrospective study of over two hundred patients from Wuhan, China, An et al. [14] reported in this issue of Digestive Diseases and Sciences that nearly a fifth of COVID-19 patients who were admitted to a hospital for quarantine had GI-COVID. The prevalence rates of anorexia, diarrhea, nausea, vomiting, and abdominal pain were similar to the numbers cited above. Liver function tests were mildly elevated in some cases but overall were similar between classical COVID-19 and GI-COVID patients. Discerning the etiology of abnormal liver function tests in COVID-19 patients remains a challenge; causes are often multifactorial and liver biopsies usually show nonspecific acute hepatitis and portal inflammation. There were no reports of severe liver dysfunction, COVID-19-related pancreatitis, or pneumatosis intestinalis in the study. Importantly, none of the GI-COVID patients developed any respiratory symptoms or fever throughout their entire length of hospital stay, and every patient in the study had a diagnosis of COVID-19 confirmed by the detection of SARS-CoV-2 RNA from polymerase chain reaction analysis of material collected by serial oropharyngeal swabs. Therefore, these findings challenge current understanding of the disease because COVID-19 is widely regarded as a respiratory infection primarily in which GI symptoms are infrequent, clustered with classical symptoms, and associated with more severe infection $[9,10]$. Also noteworthy is the observation that approximately two-thirds of the patients with GI-COVID in the study tested negative for viral RNA on their first oropharyngeal swab compared with one-third of patients with classical COVID-19 symptoms. Nevertheless, not all patients were swabbed at identical time points since atypical symptomatology affected the time to presentation to healthcare services and subsequent management. Yet, almost all patients in the study had positive IgM antibodies.

Indeed, the oro-fecal transmission of SARS-CoV-2 remains controversial. Nonetheless, An et al. [14] have reported that a small proportion of patients with GI-COVID had no lung involvement as assessed by computed tomography (CT), and furthermore, in-depth medical history of two GI-COVID clusters illustrated the transmission of disease from individuals with only GI symptoms to different individuals who later developed classical COVID-19 symptoms. Unfortunately, testing for fecal virus RNA was not attempted in the study and even if detected these observations alone cannot demonstrate causality. Interestingly, a recent systematic review and meta-analysis reported that $43 \%$ of COVID19 patients had viral shedding in their stool, which could persist for up to 70 days after the onset of symptoms [15]. Virus viability from stool samples was also demonstrated in 35\% of specifically tested patients [15]. Although current evidence is limited, such studies continue to fuel the question of whether the GI tract could serve as the primary or only site of SARS-CoV2 infection.

Another cardinal finding reported by An et al. [14] is that patients with GI-COVID took a longer time to seek healthcare services compared with typical presentations of COVID-19. Furthermore, GI-COVID patients experienced a delay in the diagnosis of COVID-19 even after evaluation by healthcare services; this was often due to misdiagnosis which had downstream effects on patient management. Therefore, given the resurgence of infection rates across the world, it is essential for clinicians to continue to exercise stringent infection control measures and to be vigilant for GI-COVID in order to limit the spread of the disease, especially in areas where the prevalence rates are high.

Lastly, two significant and potentially addressable factors that limit the insight of current systematic reviews are the lack of common standards (e.g., Bristol Stool chart to define diarrhea) and poor documentation of the time of onset and severity of GI symptoms in COVID-19 patients in the published literature. To corroborate the findings reported by An et al. [14] and provide more meaningful data for interpretation, the inclusion of hospitalized and non-hospitalized COVID-19 patient groups as well as the comprehensive detailing of symptomatology and biomarkers are recommended in future studies.

Funding This work is unfunded. JO is funded by the W D Armstrong Fellowship at the University of Cambridge and a development grant from the National University of Singapore.

\section{Compliance with Ethical Standards}

Conflict of interest The authors have none to declare.

\section{References}

1. Hoffmann M, Kleine-Weber H, Schroeder S, Krüger, et al. SARSCoV-2 cell entry depends on ACE2 and TMPRSS 2 and is blocked by a clinically proven protease inhibitor. Cell. 2020;181:271-280. e8.

2. Mandala VS, McKay MJ, Shcherbakov AA, Dregni AJ, Kolocouris A, Hong M. Structure and drug binding of the SARS$\mathrm{CoV}-2$ envelope protein transmembrane domain in lipid bilayers. 
Nat Struct Mol Biol. 2020. https://doi.org/10.1038/s41594-02000536-8.

3. Li S, Zhang Y, Guan Z, et al. SARS-CoV-2 triggers inflammatory responses and cell death through caspase-8 activation. Signal Transduct Target Ther. 2020;5:1-10.

4. Hashimoto T, Perlot T, Rehman A, et al. ACE2 links amino acid malnutrition to microbial ecology and intestinal inflammation. Nature. 2012;487:477-481.

5. Hamming I, Timens W, Bulthuis MLC, Lely AT, Navis GJ, van Goor H. Tissue distribution of ACE2 protein, the functional receptor for SARS coronavirus: a first step in understanding SARS pathogenesis. J Pathol. 2004;203:631-637.

6. Xiao F, Tang M, Zheng X, Liu Y, Li X, Shan H. Evidence for Gastrointestinal Infection of SARS-CoV-2. Gastroenterology. 2020;158:1831-1833.e3.

7. Martin TA, Wan DW, Hajifathalian K, et al. Gastrointestinal bleeding in patients with coronavirus disease 2019: a matched case-control study. Am J Gastroenterol. 2020. https://doi. org/10.14309/ajg.0000000000000805.

8. Effenberger M, Grabherr F, Mayr L, et al. Faecal calprotectin indicates intestinal inflammation in COVID-19. Gut. 2020;69:1543-1544.

9. Mao R, Qiu Y, He J-S, et al. Manifestations and prognosis of gastrointestinal and liver involvement in patients with COVID19: a systematic review and meta-analysis. Lancet Gastroenterol Hepatol. 2020;5:667-678.

10. Sultan S, Altayar O, Siddique SM, et al. AGA institute rapid review of the gastrointestinal and liver manifestations of
COVID-19, meta-analysis of international data, and recommendations for the consultative management of patients with COVID-19. Gastroenterology. 2020;159:320-334.e27.

11. Cheung KS, Hung IFN, Chan PPY, et al. Gastrointestinal manifestations of SARS-CoV-2 infection and virus load in fecal samples from a hong kong cohort: systematic review and meta-analysis. Gastroenterology. 2020;159:81-95.

12. Lin L, Jiang X, Zhang Z, et al. Gastrointestinal symptoms of 95 cases with SARS-CoV-2 infection. Gut. 2020;69:997-1001.

13. Yang X, Yu Y, Xu J, et al. Clinical course and outcomes of critically ill patients with SARS-CoV-2 pneumonia in Wuhan, China: a single-centered, retrospective, observational study. Lancet Respir Med. 2020;8:475-481.

14. An P, Chen H, Ren H, et al. Gastrointestinal symptoms onset in COVID-19 patients in Wuhan, China. Dig Dis Sci. (Epub ahead of print). https://doi.org/10.1007/s10620-020-06693-6.

15. van Doorn AS, Meijer B, Frampton CMA, Barclay ML, de Boer NKH. Systematic review with meta-analysis: SARS-CoV-2 stool testing and the potential for faecal-oral transmission. Aliment Pharmacol Ther. 2020;52:1276-1288.

Publisher's Note Springer Nature remains neutral with regard to jurisdictional claims in published maps and institutional affiliations. 